\title{
THE DRAG, LIFT AND STROUHAL NUMBER OF A CIRCULAR-SECTION CYLINDER WITH A CONIC DISTURBANCE AT SUBCRITICAL REYNOLDS NUMBERS
}

\author{
Liming Lin, Xingfu Zhong, Yingxiang Wu \\ The Key Laboratory for Mechanics in Fluid Solid Coupling Systems \\ Institute of Mechanics \\ Chinese Academy of Sciences \\ Beijing, China 100190 \\ Email: Ilmbirthday@163.com
}

\begin{abstract}
Through numerical simulations, the flow past a circularsection cylinder with a conic disturbance is investigated at subcritical Reynolds numbers of $10^{2}, 10^{3}$ and $10^{4}$. At higher Reynolds numbers, LES is employed in simulating the turbulence transition in the near wake. Computations with a series of parameters, the wavelength and wave steepness, are carried out. Generally, the drag of conic cylinder is gradually increased with the increasing wave steepness and in most cases greater than that of the cylinder without disturbance. However, the total lift in most cases is obviously reduced. The phenomenon of local minimum is found out in variation of drag and lift. The introduction of conic disturbance also leads to the decrease of frequency of vortex shedding as the wave steepness increases.
\end{abstract}

Keywords: Drag, lift, Strouhal number, conic disturbance, circular cylinder, subcritical Reynolds numbers.

\section{INTRODUCTION}

Bluff bodies are commonly used in many engineering applications, such as mooring cables, petroleum production risers, pipelines in marine technology, buildings and suspension bridges. The unsteady wake with the vortex shedding alternatively can generate the unsteady fluid forces. The structure is subject to excitation and therefore vibrated, called the vortexinduced vibration (VIV). When the frequency of vortex shedding is gradually close to the natural frequency of the body, the lock- in occurs with suddenly amplified fluid forces and structural oscillating amplitude. It results in the fatigue damage, having the destructive potential for the structural integrity, even the safety of people and production. In a half century, many studies have been reported in investigating and understanding the dynamics of VIV. Comprehensive reviews can be referenced in [1, 2, 3].

Over recent several decades, a large number of methods have been proposed in order to disturb and control the flow around the body. It leads to weaken, even totally suppress, the vortex shedding, as well as the fluctuating amplitude of fluid forces. For example, the surface control bump was installed spirally around the body [4]. Drag reduction of $47 \%$ was reached. At a certain wave steepness, defined by the ratio of the wave height to the wavelength, there was no sign of unsteady vortex shedding. However, such device was mainly effective in the great massdamping parameter. Two small rotating cylinders were arranged in the boundary layer of the cylinder, delaying the separation of the boundary layer [5]. The drag reduction was almost $60 \%$. As a passive method, triple-starting helical grooves and multiple control rods were recently applied [6, 7|. Until now, streamline fairing [8] still exhibits a very good aerodynamic performance owing to streamlined outer shape, resulting in the delay of the flow separation, similarly as for splitter plates [9]. Because of variable ocean flow direction, the rotatable device is usually installed. This could introduce a new dynamical instability. Helical strake [10], the most widely used presently, is a device in disturbing spanwise uniformity of vortex shedding. More details 


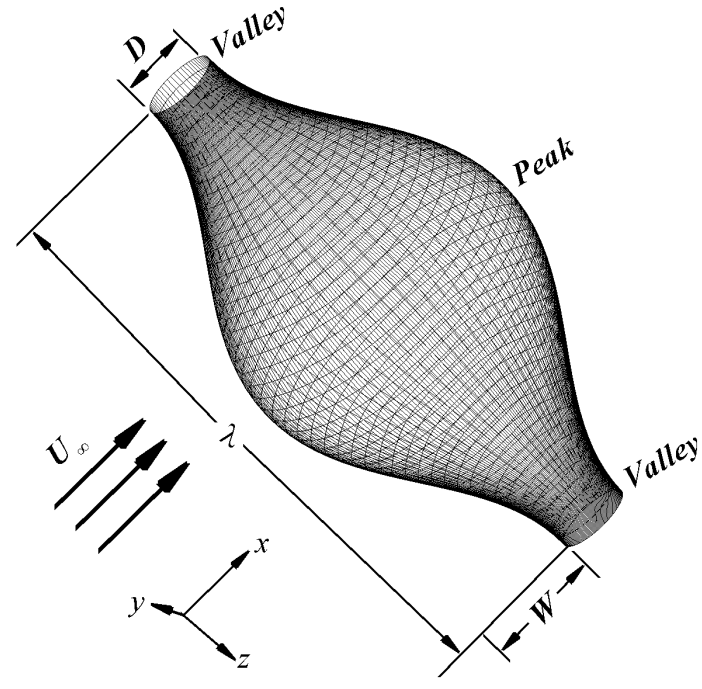

(a)

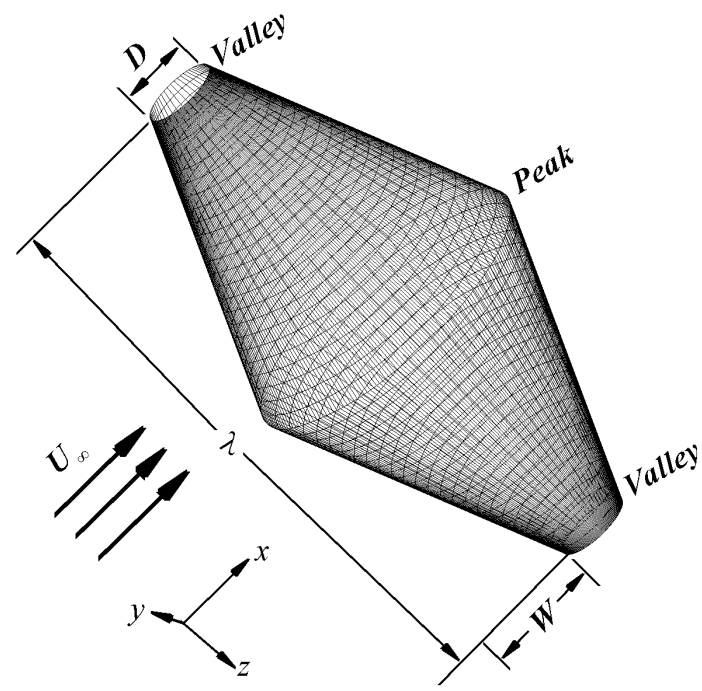

(b)

FIGURE 1. SCHEMATICS OF FLOWS PAST A CIRCULARSECTION CYLINDER WITH (a) THE HARMONIC AND (b) CONIC DISTURBANCE ALONG THE SPAN.

on other devices can be found out in previous review [11].

On the other hand, three-dimensional geometric disturbance, such as a wavy front surface [12] and wholly wavy cylinder [13, 14], was introduced as another VIV suppressing method. Considering the sensitivity to the flow direction, a new idea that disturbance was introduced in a radial-spanwise plane, called radial disturbance, was proposed [15, 16]. As a fundamental study, experiments for oscillating pendulum with two types of radial disturbances in water channel were performed. The structural oscillating amplitude at the start of synchronization was really reduced but increased subsequently at high velocity. Owing to obvious difference between pendulum and riser, the flow around the fixed cylinder with circular-section and two types of radial disturbances, harmonic and conic, as shown in Fig 1, was investigated at $R e=100$ [17, 18]. The introduction of disturbances leads to the original vortex pattern becoming more complex, such as the $\Omega$-type vortex, even the complete suppression of Kármán's vortex street. But even so, there are still some unknown issues to be explored, especially at higher Reynolds numbers.

As a part of series of works, the main purpose at present paper is to get to know and compare the effect of radial disturbance on hydrodynamic parameters, e.g. coefficients of drag and lift and frequency of vortex shedding, through the numerical simulation at $R e=10^{2}, 10^{3}$ and $10^{4}$. There is much of similarity between harmonic and conic disturbances, typically in the vortex pattern in the parameter space as shown in Fig.2. Only conic disturbance is thus taken into account here with a series of wavelength and wave steepness. Firstly, the physical model is described briefly. Then effects of disturbances on the drag, lift and frequency of vortex shedding and so on, are presented and analyzed, respectively. And conclusions are made at last.

\section{PHYSICAL MODEL \\ Governing Equations}

As shown in Fig 1) (b), we consider the motion of a viscous fluid past a circular-section cylinder with the conic disturbance along the span, which is placed perpendicular to a uniform free stream. Such cylinder is referred to as the conic cylinder, different from the straight cylinder with the constant diameter. The peak and valley of the conic cylinder are defined as the spanwise positions with the maximal and minimal diameters, respectively. The wavy shape can be mathematically described by the wavelength $\lambda$, the peak-to-valley wave height $W$ and the base diameter $D$, equal to the diameter of valley. Therefore, the diameter of peak is $2 W+D$. For the sake of applying such disturbance as a suppression shroud in pre-designed flexible pipelines or risers, the diameter of the straight cylinder must be equal to $D$. In present inertial Cartesian coordinate system, the $x$-axis (streamwise direction) is aligned with the free-stream velocity $U_{\infty}$, the $y$ axis (vertical direction) is normal to the free stream and the $z$-axis (spanwise direction) is along with the span of the cylinder. The fluid is assumed to have constant density $\rho$ and dynamic viscosity $\mu$, then the constant kinematic viscosity $v=\mu / \rho$. The nondimensional control parameter for such idealized incompressible flow is defined as the Reynolds number, $R e=U_{\infty} D / v$.

The evolution of the flow is described by the incompressible continuity and Navier-Stokes equations in non-dimensional 


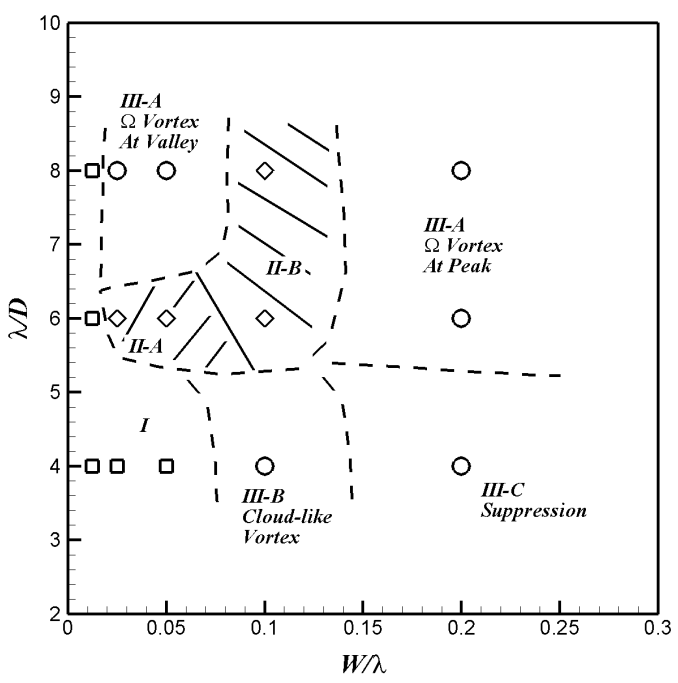

(a)

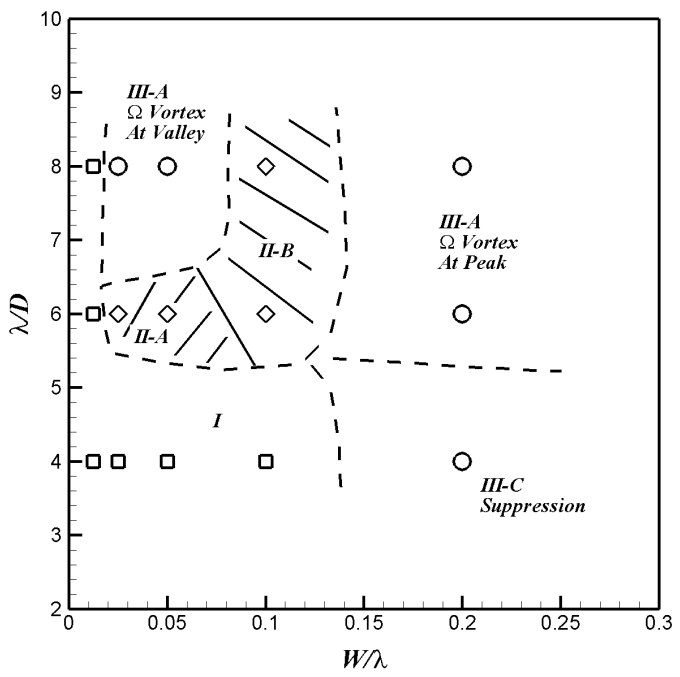

(b)

FIGURE 2. SUMMARY OF THE WAVELENGTH $\lambda / D$ VERSUS THE WAVY STEEPNESS $W / \lambda$ FOR ALL COMPUTATIONAL CASES OF (a) THE HARMONIC AND (b) CONIC CYLINDERS [17], WHERE $\square$ DENOTES THE REGIME I OF WEAK DISTURBANCE, $\diamond$ THE REGIME II OF MODERATE DISTURBANCE AND $\circ$ THE REGIME III OF STRONG DISTURBANCE. THE DASHED LINES ARE JUST THE BOUNDARY LINES TO SEPARATE DIFFERENT REGIMES. THE DASHED REGION WITH OBLIQUE LINES DENOTES APPROXIMATELY THE OCCURRENCE OF REGIME II.

forms written as

$$
\begin{aligned}
\nabla \cdot \mathbf{u} & =0 \\
\frac{\partial \mathbf{u}}{\partial t}+(\mathbf{u} \cdot \nabla) \mathbf{u} & =-\nabla p+\frac{1}{R e} \nabla^{2} \mathbf{u}
\end{aligned}
$$

where $\mathbf{u}=(u, v, w)$ is the velocity vector, $p$ is the static pressure, $t$ is the non-dimensional time and $\nabla$ is the gradient operator. In the periodic flow, the frequency of vortex shedding, $f$, is nondimensionalized as the Strouhal number, that is $S t=f D / U_{\infty}$. Lengths are scaled by the cylinder base diameter $D$ and velocities by the free-stream velocity $U_{\infty}$.

Due to the laminar flow at $R e=100$, DNS is applied. However, at higher Reynolds numbers, the large eddy simulation (LES) is adopted in simulating turbulent wake transition.

\section{Numerical Method}

The simulated flow would be carried out with proper boundary conditions. In order to approximatively simulate the infinite length of cylinder, the periodic flow along the span is assumed. Then only boundary conditions in $(x, y)$ plane needs to be described. At inlet of computational domain, the uniform free-stream velocity is prescribed. The non-reflecting outflow is adopted at outlet. At vertical sides of computational domain, the free slip is applied. On cylinder surfaces, non-slip boundary condition is used.

Due to the usage of structural grids in a hexagonal form, the computational domain in $(x, y)$ plane is just presented as shown in Fig 3 . The non-dimensional size is $40 \times 20(x \times y)$. The dimensionless computational length in the spanwise direction is given as one period of wavy disturbance, equal to $\lambda / D$, as well as for the straight cylinder. The grids of 17600 in $(x, y)$ plane are mainly concentrated near the cylinder surface and the near wake, and coarse in the remaining region. The grid in $z$-axis is 5 per unit length. Although the refinement study in grids as the fundamental work, mainly for the straight cylinder, is not given here, the hydrodynamic parameters and vortex pattern are presented in the following context.

A non-dimensional time step of 0.01 was used with secondorder time discretization. The maximal magnitude of convergence error of $10^{-3}$ is obtained for the continuity equation.

Simulation runs are performed by the Fluent. The SIMPLEC algorithm is selected for the pressure-velocity coupling. The second-order discretization is applied for the pressure and the bounded central differencing scheme for the momentum. The $\mathrm{W}$-cycle multiple-grid method is adopted in solving pressure.

\section{RESULTS}

In contrast to the typical flow past the straight cylinder, two extra length scales $W$ and $\lambda$ are introduced as for the conic cylinder. As a result, a further two independent length parameters, the non-dimensional wavelength $\lambda / D$ and the wave steepness $W / \lambda$, can be obtained in describing the similarity between different conic cylinders. There is a large three-dimensional parameter space based upon $\operatorname{Re}, W / \lambda$ and $\lambda / D$. A series of computational investigations have been carried out at subcritical Reynolds num- 


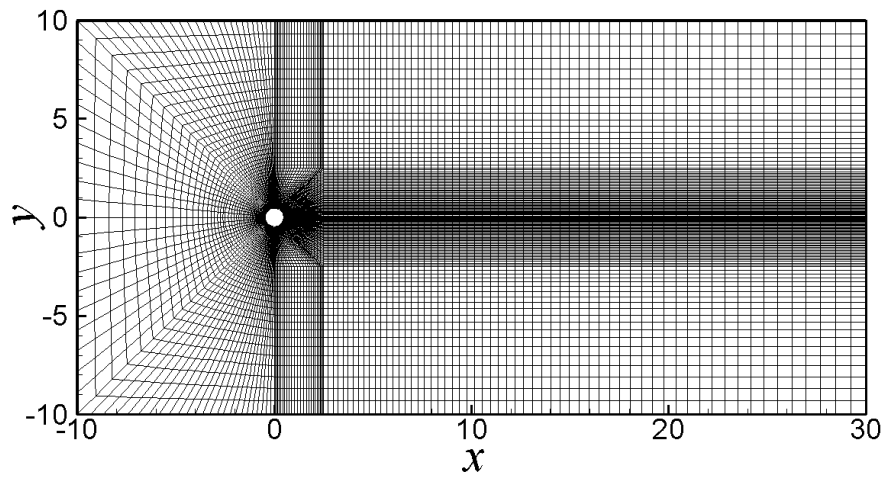

FIGURE 3. NON-DIMENSIONAL COMPUTATIONAL DOMAIN AND GRIDS IN $(x, y)$ PLANE.

bers of $10^{2}, 10^{3}$ and $10^{4}$ for different $W / \lambda(0.0125,0.025,0.05$, $0.1,0.2)$ and $\lambda / D(4,6,8)$ with constant diameter $D$. So, the straight cylinder can also be obtained as the wave steepness $W / \lambda$ reaches down to zero and is only varied with $\lambda / D$.

\section{Straight Cylinder}

As a basic case, the flow around the straight cylinder is calculated at subcritical Reynolds numbers. At $R e=100$, the twodimensional wake topology is typically identified as the wellknown Kármán vortex, as shown in Fig 4 (a). With the increasing Reynolds number, the instability and turbulent transition are occurred in the near wake. The most interesting feature of the flow is three-dimensional structure with the generation of additional components of vorticity, $\omega_{x}$ and $\omega_{y}$. Figures 4 (b) and (c) show a visualization of the fully evolution of the vortex pattern at $R e=10^{3}$ and $10^{4}$ and $\lambda / D=8$. The distorted spanwise vortex pairs are still alternatively shedding accompany with the staggered array of streamwise vortices at a higher wavenumber.

As shown in Tab 1 , the mean drag coefficient, $C_{\mathrm{D}_{-} \mathrm{M}}$, and the Strouhal number, $S t$, at different Reynolds numbers and spanwise length are well compared with the previous experiments [19]. The difference in drag, less than $10 \%$, in present simulations at moderate Reynolds numbers is mainly attributed to the variation of spanwise length of cylinder and complex vortex patter in the near wake, as well as the lift in the following context.

\section{Drag}

The results for the variation of the mean drag coefficient $C_{\text {D_M }}$ with respect to the non-dimensional wavelength $\lambda / D$ and the wave steepness $W / \lambda$ at different Reynolds numbers are summarized in Fig 5 . Note that the drag, is normalized by the freestream dynamic pressure and the projected area of the body to produce the drag coefficient, $\frac{1}{2} \rho U_{\infty}^{2} A$, as well as the lift and lift coefficient in the next context. For the sake of comparison with

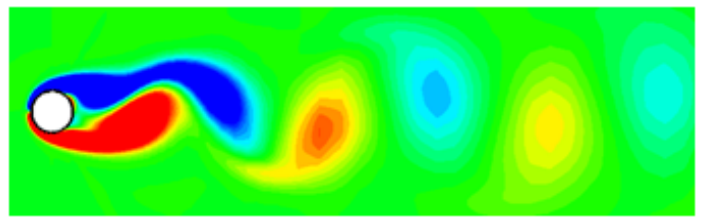

(a)

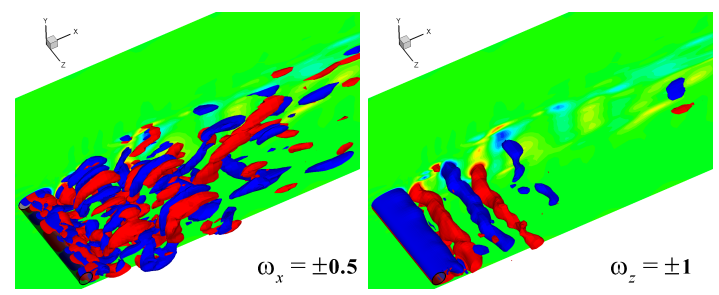

(b)

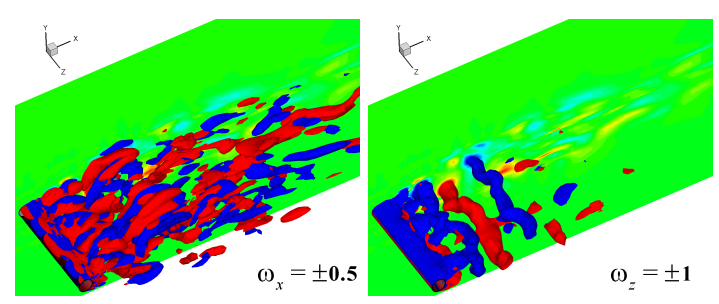

(c)

FIGURE 4. AS FOR THE BASE CASE, CONTOURS OF (a) THE SPANWISE VORTICITY IN DESCRIBING THE Kármán VORTEX SHEDDING AT $R e=100$; STREAMWISE VORTICITY $\omega_{x}= \pm 0.5$ (RED/BLUE) AND SPANWISE VORTICITY $\omega_{z}= \pm 1$ (RED/BLUE) IN DESCRIBING THREE-DIMENSIONAL WAKE AT (b) $R e=10^{3}$ AND $\lambda / D=8$, AND (c) $R e=10^{4}$ AND $\lambda / D=8$. THE SLICE AT $z=0$ IS THE CONTOUR OF $\omega_{z}$. NOTE THAT THE CYLINDER IS SHOWN BY USING A WIRE FRAME.

the straight cylinder, such projected area, $A$, must be computed from the straight cylinder with the same spanwise length, that is $A=\lambda D$, instead of the conic cylinder.

At the laminar flow of $R e=10^{2}$, the introduction of conic disturbance leads to the greater drag, compared to that of the straight cylinder. Generally, as the wave steepness $W / \lambda$ increases, the drag is almost linearly increased with the certain wavelength $\lambda / D$. Especially an interesting phenomenon is occurred in the case of $W / \lambda=0.025$ and $\lambda / D=6$, where the drag reaches a local minimum. On the other hand, at the same $W / \lambda$ greater than 0.05 , the drag is also increased with the increasing $\lambda / D$. At small values of $W / \lambda$ less than 0.05 , the drag of the conic cylinder with $\lambda / D=4$ is nearly close to that with $\lambda / D=8$.

As the Reynolds number reaches up to $10^{3}$, the phenomenon of local minimum is also found out for all computed wavelengths within the range of $W / \lambda$ from 0.0125 to 0.025 . The drag reductions are $2.3 \%, 7.3 \%$ and $9.4 \%$ for the wavelength of $\lambda / D$ of 4 , 
TABLE 1. COMPARISON OF THE MEAN DRAG AND STROUHAL NUMBER BETWEEN PRESENT COMPUTATIONS AND PREVIOUS EXPERIMENTAL RESULTS.

\begin{tabular}{lcll}
\hline & & $C_{\mathrm{D} \_\mathrm{M}}$ & $S t$ \\
\hline$R e=10^{2}$ & Present & 1.39 & 0.165 \\
& Experiment & $1.25 \sim 1.4 ; 1.8$ & 0.164 \\
$R e=10^{3}$ & Present & $1.27 \sim 1.31$ & $0.197 \sim 0.201$ \\
& Experiment & 1 & 0.21 \\
$R e=10^{4}$ & Present & $1.356 \sim 1.473$ & $0.173 \sim 0.18$ \\
& Experiment & 1.2 & $0.19 \sim 0.205$ \\
\hline
\end{tabular}

6 and 8 , respectively. When the wave steepness $W / \lambda$ is greater than 0.025 , the drag is also linearly increased. And the drag with the wavelength of 4 is almost equal to that with $\lambda / D=6$.

Similarly at $R e=10^{4}$, the local minimum is occurred with $W / \lambda \leq 0.05$ for different $\lambda / D$. Correspondingly, the drag reductions of $8.1 \%, 11.4 \%$ and $5 \%$ are obtained for $\lambda / D$ of 4,6 and 8 , respectively. Then the drag is always increased as the wave steepness $W / \lambda$ increases greater than 0.05 .

\section{Lift}

The magnitude of the root mean square (RMS) of the total lift coefficient $C_{\mathrm{L}_{-} \mathrm{RMS}}$ is commonly used as an indicator of the level of unsteadiness in the near wake flow. The results for the variation of $C_{\mathrm{L} \_\mathrm{RMS}}$ with respect to the non-dimensional wavelength $\lambda / D$ and the wave steepness $W / \lambda$ at different Reynolds numbers are summarized in Fig 6

As for $R e=10^{2}$, the lift in most cases, except for $\lambda / D=4$, is obviously less than that of the straight cylinder. At $\lambda / D=4$, the lift is firstly increased with the increasing $W / \lambda$ until 0.1 to reach up to the local maximum, and then reduced quickly down to zero with $W / \lambda=0.2$, in which the complete suppression of the Kármán vortex street is observed. For other cases with $\lambda / D=6$ and 8 , as $W / \lambda$ increases, the lift decreases rapidly at the beginning until $W / \lambda$ reaches in the range from 0.025 to 0.05 , then increases slowly at $W / \lambda=0.1$, and finally reduces down to near zero. Therefore, similar to variation of drag, the local minimum of the lift is appeared near $W / \lambda=0.05$. The reductions of lift are $76.6 \%$ and $68.8 \%$ for $\lambda / D=6$ and 8 , respectively.

At the Reynolds number increasing up to $10^{3}$, with the increasing $W / \lambda$, the lift is quickly reduced at first until 0.05 , and then increases slowly. Especially for the case of $\lambda / D=6$, the lift reduces again after reaches the local maximum at $W / \lambda=0.1$. Analogously, the local minimum of lift is obtained near $W / \lambda=$

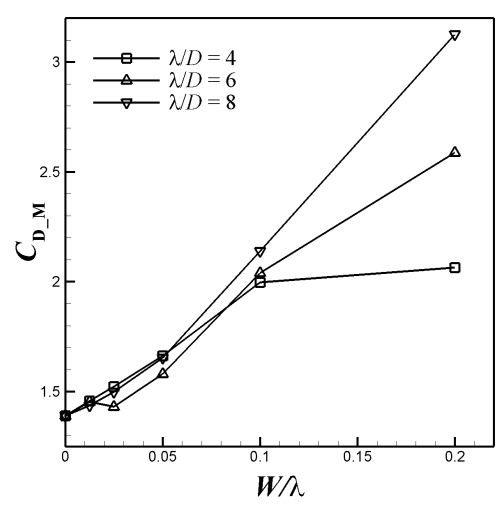

(a)

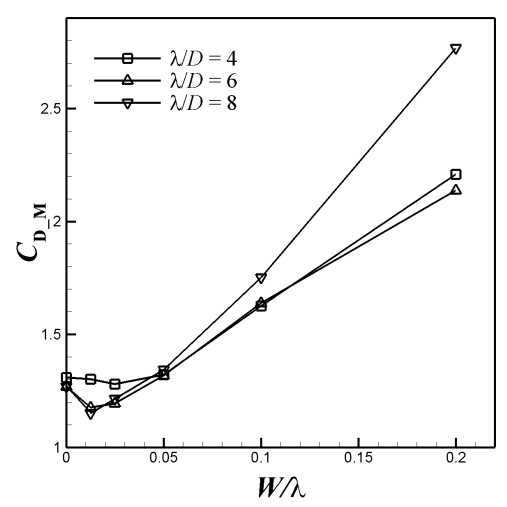

(b)

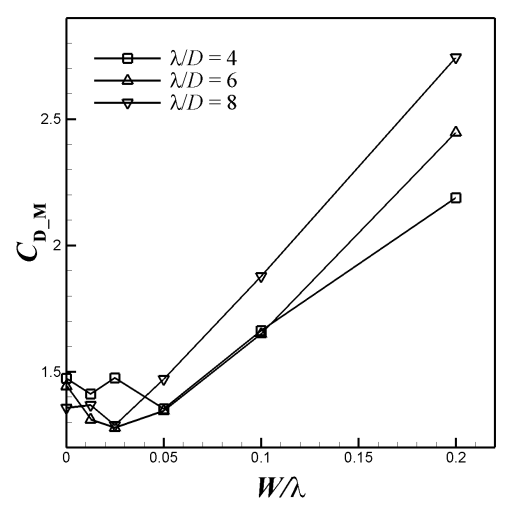

(c)

FIGURE 5. VARIATION OF THE MEAN DRAG COEFFICIENT $C_{\text {D_M }}$ WITH RESPECT TO THE NON-DIMENSIONAL WAVELENGTH $\lambda / D$ AND THE WAVE STEEPNESS $W / \lambda$ AT REYNOLDS NUMBERS OF (a) $10^{2}$, (b) $10^{3}$ AND (c) $10^{4}$.

0.05. And the lift reductions are $60.3 \%, 81.4 \%$ and $91.9 \%$ for $\lambda / D=4,6$ and 8 , respectively.

With the Reynolds number of $10^{4}$, the variation of lift behaves in a similar way at $R e=10^{3}$. Furthermore, the local mini- 


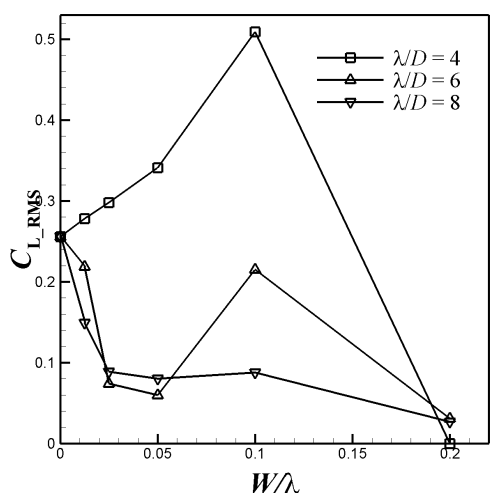

(a)

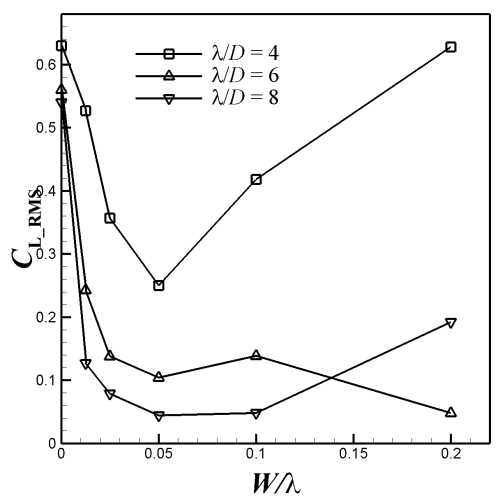

(b)

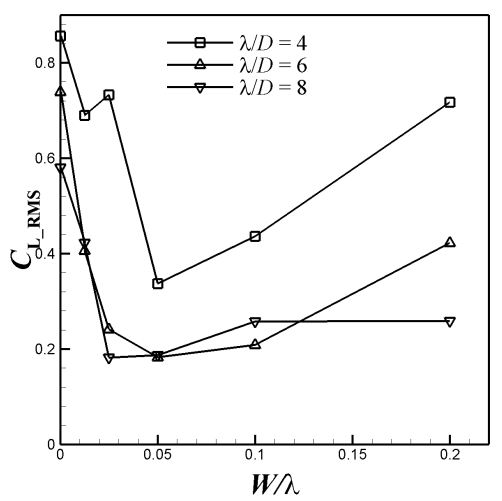

(c)

FIGURE 6. VARIATION OF THE RMS LIFT COEFFICIENT $C_{\text {L_RMS }}$ WITH RESPECT TO THE NON-DIMENSIONAL WAVELENGTH $\lambda / D$ AND THE WAVE STEEPNESS $W / \lambda$ AT REYNOLDS NUMBERS OF (a) $10^{2}$, (b) $10^{3}$ AND (c) $10^{4}$.

mum is occurred with the wave steepness less than 0.05. Correspondingly, the lift reductions are obtained as $60.6 \%, 75.2 \%$ and $68.2 \%$ for $\lambda / D=4,6$ and 8 , respectively. In particular, when $W / \lambda$ increases from 0.1 to 0.2 , the lift is almost invariant for $\lambda / D=8$, while increases for other wavelength.

In summary, the local minimum for the drag and lift gives us a suggestion that there exists the optimized range for specific $\lambda / D$ and $W / \lambda$ in initial design by the usage of conic disturbance. In such region of parameter space, the drag could be reduced or increased a little, while the lift is reduced greatly.

\section{Frequency of vortex shedding}

Figure 7 presents the variation of non-dimensional frequency of vortex shedding, $S t$, obtained from the time history of total lift, with respect to the wave steepness $W / \lambda$ and wavelength $\lambda / D$. At present Reynolds numbers, the frequency is generally reduced as the wave steepness increases. And in most cases at the same wave steepness, the frequency with the greater wavelength is less than that with the smaller wavelength.

On the other hand, the characteristics of vortex shedding frequency varied along the time could be investigated by the Morlet wavelet analysis [20]. In the flow past the straight cylinder at present Reynolds numbers, the near wake can still be identified by the alternative vortex shedding. Therefore, the frequency is mainly singular in the whole computational time, whatever for the higher or lower level of the peak in the time history of the oscillating lift coefficient, typically as shown in Fig 8 . After introducing the conic disturbance, with the appearance of the more and more complex vortex pattern in the near wake, such as the $\Omega$ type vortex shed at the valley at the Reynolds number of $10^{2}[17]$, the frequency could not be always kept a single one. And two or three frequencies are distinguished with special features, related with the modification of fluctuating amplitude of lift along the time. At higher Reynolds numbers, there are also multiple frequencies in some cases. Typically as shown in Fig 9 (a), the appearance of mixed multiple frequencies is associated with the increasing amplitude of lift, while only main frequency exists on the lower level of lift amplitude. In other situation as shown in Fig 9 (b), the second frequency is sometimes appeared in certain period with the lower peak of lift. Specially at the Reynolds number of $10^{3}$, a very lower frequency is identified, sometimes throughout the whole computation, as shown in Fig 10

\section{Vortex pattern in the near wake}

In the flow around the bluff body, the vorticity is mainly generated on the body's surface due to the viscosity and then transferred into the near wake. The introduction of conic disturbance directly results in the spanwise flow disrupted through the nonslip boundary condition. For example, the flow past the straight cylinder at $R e=10^{2}$ is two-dimensional. The three-dimensional effect of the conic disturbance leads to the specific distribution of additional components of surface vorticity, and the obvious three-dimensionality of the near wake flow. Different vortex patters are classified based on the extent of transformation of original Kármán vortex, as reported in previous work [17]. Appar- 


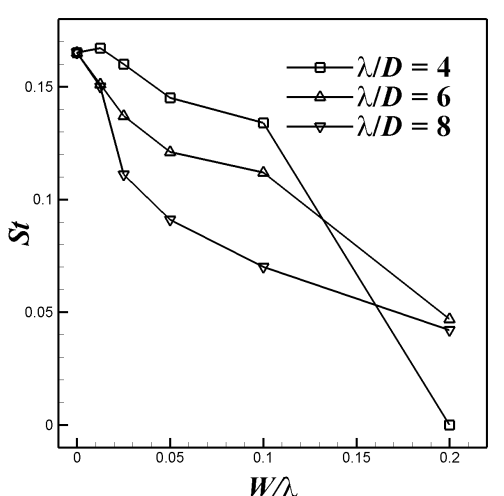

(a)

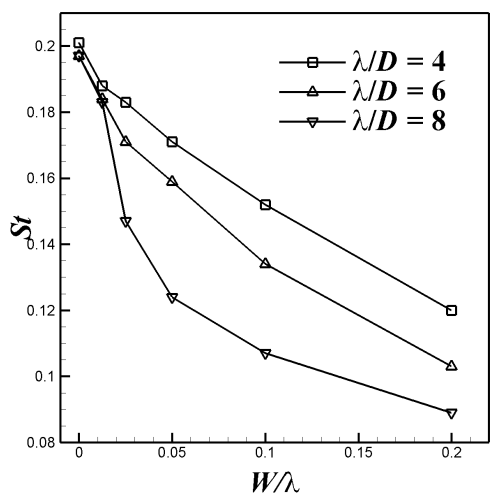

(b)

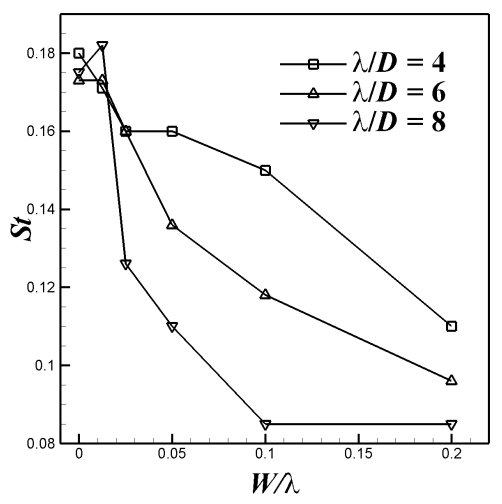

(c)

FIGURE 7. VARIATION OF THE STROUHAL NUMBER $S t$ WITH RESPECT TO THE NON-DIMENSIONAL WAVELENGTH $\lambda / D$ AND THE WAVE STEEPNESS $W / \lambda$ AT REYNOLDS NUMBERS OF (a) $10^{2}$, (b) $10^{3}$ AND (c) $10^{4}$.

ently, as shown in Fig 4 strong and natural three-dimensionality of the flow past the straight cylinder at higher Reynolds numbers is associated with the complex distribution of additional components of vorticity. It makes hardly to find out the effect of the

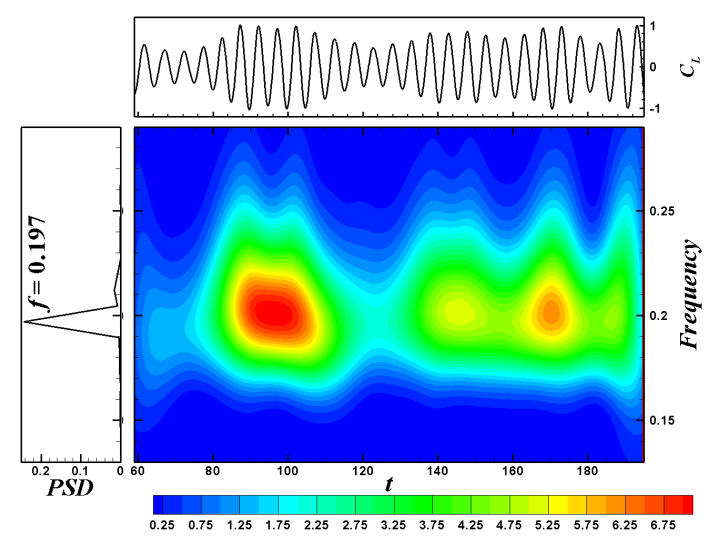

FIGURE 8. THE FREQUENCY AND THE MORLET WAVELET ANALYSIS FOR THE FLOW PAST THE STRAIGHT CYLINDER AT THE NON-DIMENSIONAL WAVELENGTH $\lambda / D$ OF 8 AND THE REYNOLDS NUMBER OF $10^{3}$.

conic disturbance on vortex pattern. Interestingly, two patterns, $\Omega$-type vortex shed at the valley and peak, similar to those appeared in strong effect of disturbance at $R e=10^{2}$, as shown in Fig 11, are identified at different wave steepness at the wavelength of 6 and the Reynolds number of $10^{3}$.

Because the discussion on the effect of conic disturbance on the vortex pattern in the near wake is not one of main objects in present paper, further study would be reported in future.

\section{CONCLUSIONS}

Through numerical simulations, the flow past the circularsection cylinder with the conic disturbance is investigated at subcritical Reynolds numbers of $10^{2}, 10^{3}$ and $10^{4}$. A series of control parameters for different wavy geometric disturbance are carried out for the non-dimensional wavelength $\lambda / D$ of 4,6 and 8 and the wave steepness $W / \lambda$ of $0.0125,0.025,0.05,0.1$ and 0.2 . In present paper, the variation of main hydrodynamic parameters, coefficients of drag and lift and the frequency of vortex shedding, are obtained with respect to the wavelength and wave steepness. Characteristics are summarized as follows:

(1) With the increasing wave steepness, the drag generally increases. At lower wave steepness less than 0.05, there exists the phenomenon of the local minimum of drag. Sometimes, it would be obviously less than the drag of the straight cylinder. The maximum of drag reduction reaches up to $11.4 \%$ at $R e=10^{4}$, $\lambda / D=6$ and $W / \lambda=0.025$.

(2) However, the total lift in most cases is firstly reduced quickly as the wave steepness increases, and then increases slowly. In some cases, especially at $R e=100$, the lift is reduced near zero at $W / \lambda=0.2$. Similarly, the local minimum is also appeared at lower wave steepness. The maximum of lift reduction is $91.9 \%$ 


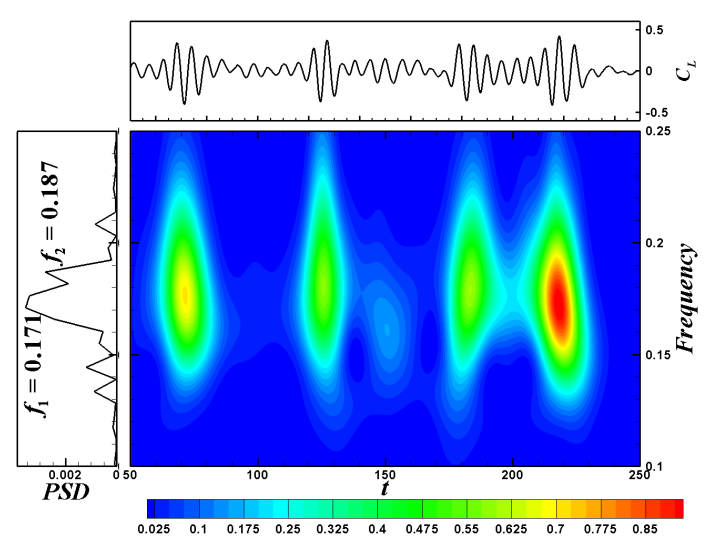

(a)

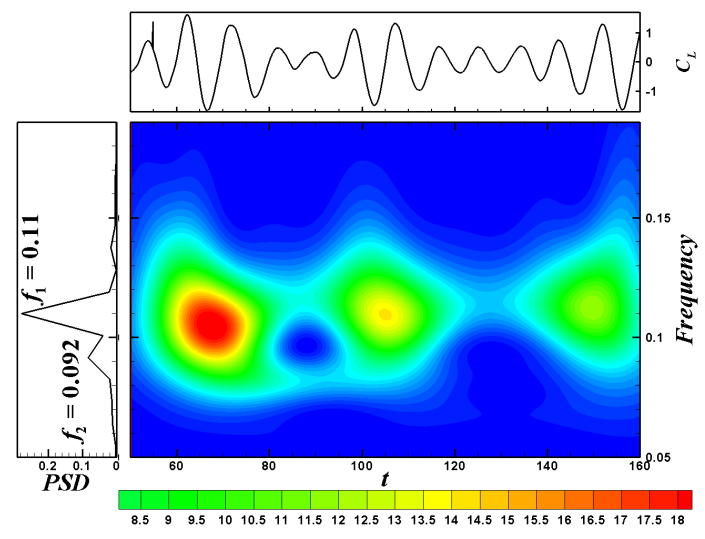

(b)

FIGURE 9. THE FREQUENCY AND THE MORLET WAVELET ANALYSIS FOR THE FLOW PAST THE CONIC CYLINDER AT (a) $\lambda / D=6, W / \lambda=0.025$ AND $R e=10^{3}$ AND (b) $\lambda / D=4, W / \lambda=0.2$ $\mathrm{AND} R e=10^{4}$.

at $W / \lambda=0.05, \lambda / D=8$ and $R e=10^{3}$.

(3) At present Reynolds numbers, the introduction of disturbance leads to the frequency of vortex shedding in most cases reduced with the increasing wave steepness. Through the wavelet analysis, multiple frequencies are identified and varied with the higher or lower level of peak amplitude of lift coefficient. Furthermore, such property is related to the complicated vortex pattern in the near wake, such as the $\Omega$-type vortex shed at the valley or peak.

\section{ACKNOWLEDGMENT}

The authors sincerely acknowledge the support of the National Key Scientific Instrument and Equipment Development Program of China (No. 2011YQ120048) for the present work.

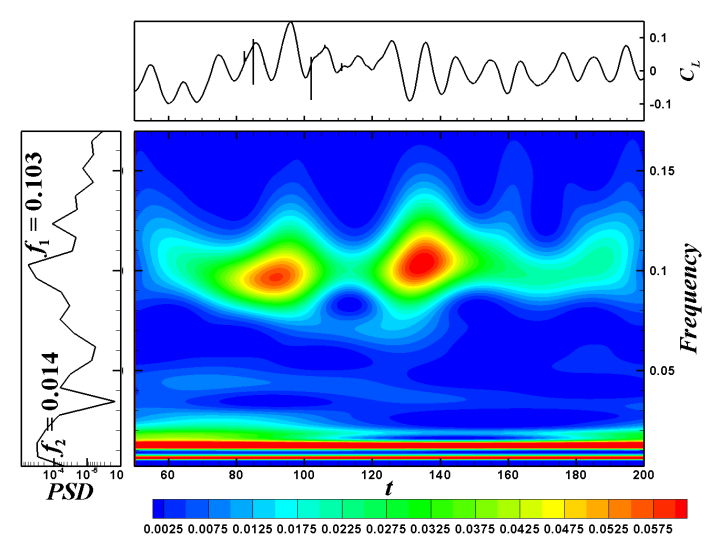

FIGURE 10. THE FREQUENCY AND THE MORLET WAVELET ANALYSIS FOR THE FLOW PAST THE CONIC CYLINDER AT $\lambda / D=6, W / \lambda=0.2$ AND $R e=10^{3}$.
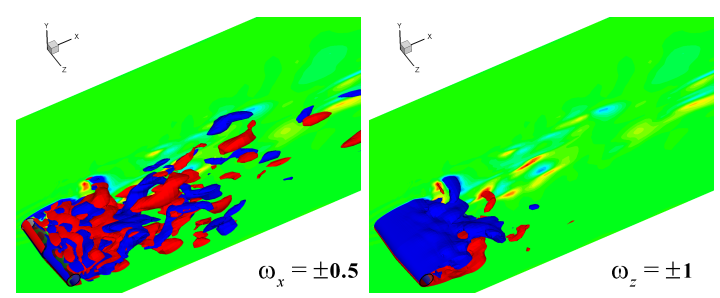

(a)

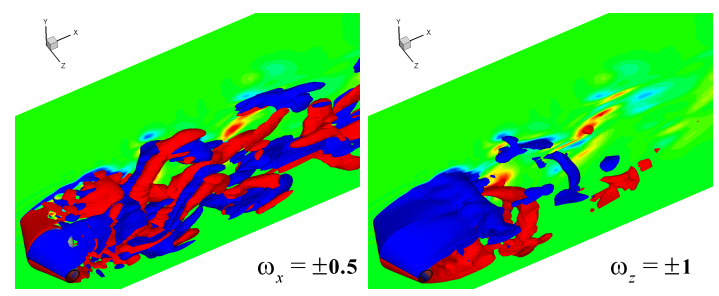

(b)

FIGURE 11. CONTOURS OF STREAMWISE VORTICITY $\omega_{x}=$ \pm 0.5 (RED/BLUE) AND SPANWISE VORTICITY $\omega_{z}= \pm 1$ (RED/BLUE) IN DESCRIBING THREE-DIMENSIONAL WAKE AT (a) $W / \lambda=0.025$ AND (b) $W / \lambda=0.2$ WITH $\lambda / D=6$ AND $R e=10^{3}$.

\section{REFERENCES}

[1] Sarpkaya, T. A critical review of the intrinsic nature of vortex-induced vibrations. Journal of Fluids and Structures, 2004, Vol.19, pp.389-447.

[2] Williamson, C.H.K. and Govardhan, R. Vortex-induced vibrations. Annual Review of Fluid Mechanics, 2004, Vol.36, pp.413-455.

[3] Gabbai, R.D. and Benaroya, H. An overview of modeling and experiments of vortex-induced vibration of circular cylinders. Journal of Sound and Vibration, 2005, Vol.282, pp.575-616. 
[4] Owen, J.C., Bearman, P.W. and Szewczyk, A.A. Passive control of VIV with drag reduction. Journal of Fluids and Structures, 2001, Vol.15, pp.597-605.

[5] Korkischko, I. and Meneghini, J.R. Suppression of vortexinduced vibration using moving surface boundary-layer control. Journal of Fluids and Structures, 2012, Vol.34, pp.259-270.

[6] Huang, S. VIV suppression of a two-degree-of-freedom circular cylinder and drag reduction of a fixed circular cylinder by the use of helical grooves. Journal of Fluids and Structures, 2011, Vol.27, pp.1124-1133.

[7] Wu, H., Sun, D.P. and Lu, L. et al. Experimental investigation on the suppression of vortex-induced vibration of long flexible riser by multiple control rods. Journal of Fluids and Structures, 2012, Vol.30, pp.115-132.

[8] Lee, L. and Allen, D.W. The dynamic stability of short fairings. Offshore Tech Conf Houston, Texas, USA, 2005, OTC-17125.

[9] Assi, G.R.S., Bearman, P.W. and Kitney, N. Low drag solutions for suppressing vortex-induced vibration of circular cylinders. Journal of Fluids and Structures, 2009, Vol.25, pp.666-675.

[10] Korkischko, I. and Meneghini, J.R. Experimental investigation of flow-induced vibration on isolated and tandem circular cylinders fitted with strakes. Journal of Fluids and Structures, 2010, Vol.26, pp.611-625.

[11] Kumar, R.A., Sohn, C.H. and Gowda, B.H.L. Passive control of vortex-induced vibrations: an overview. Recent Patents on Mechanical Engineering, 2008, Vol.1, pp.1-11.

[12] Bearman, P.W. and Owen, J.C. Reduction of bluff-body drag and suppression of vortex shedding by the introduction of wavy separation lines. Journal of Fluids and Structures, 1998, Vol.12, pp.123-130.

[13] Owen, J.C., Szewczyk, A.A. and Bearman, P.W. Suppressing Kármán vortex shedding by use of sinuous circular cylinders. Bulletin of the American Physical Society, 1999, Vol.44, pp.124.

[14] Lin, L.M., Ling, G.C. and Wu, Y.X. Mechanism responsible for the complete suppression of Karman vortex in flows past a wavy square-section cylinder. Chinese Physics Letters, 2010, Vol.27, pp.034702.

[15] Lin, L.M., Zhong, X.F. and Wu, Y.X. Experimental investigation of a new device in suppressing vortex-induced vibrations of a circular cylinder. Proc. 21st Int. Offshore and Polar Eng. Conf., Maui, Hawaii, ISOPE, 2011, pp.12831288.

[16] Lin, L.M., Zhong, X.F. and Wu, Y.X. Vortex-induced vibrations of a circular cylinder with different geometric disturbances. Proc. 22nd Int. Offshore and Polar Eng. Conf., Rhodes, Greece, ISOPE, 2012, pp.623-629.

[17] Lin, L.M., Zhong, X.F. and Wu, Y.X. Flow around a circular cylinder with radial disturbances at a low Reynolds number. Proc. 23rd Int. Offshore and Polar Eng. Conf., Anchorage, Alaska, USA, ISOPE, 2013, pp.387-394.

[18] Lin, L.M., Zhong, X.F. and Wu, Y.X. Characteristics for a flow past a circular cylinder with two types of radial disturbances at $\mathrm{Re}=100$. Advanced Materials Research, 2014, Vol.871, pp.107-114.

[19] Sarpkaya, T. and Isaacson, M. Mechanics of wave forces on offshore structures. Van Nostrand Reinhold Company, New York, USA, 1981.

[20] Jaideva, C.G. and Andrew, K.C. Fundamentals of wavelets: theory, algorithms, and applications. Wiley series in microwave and opticaw engineering, New Jork: A Wileyinterscience publication, John Wiley \& Sons, INC., 1999. 